\title{
Editorial to the Inaugural Volume of the Journal of Well-Being Assessment
}

\author{
Ingrid Brdar $^{1} \cdot$ René Proyer $^{2} \cdot$ Veljko Jovanović $^{3}$
}

Published online: 30 May 2018

(C) Springer International Publishing AG, part of Springer Nature 2018

We are pleased to announce the inaugural volume of the Journal of Well-Being Assessment, a new journal dedicated to the advancement of the well-being assessment theory, research and practice across domains and cultures. The journal aims at including different assessment topics and theories within the common topic of well-being. A new journal on well-being assessment not only brings together separate fields of assessment and well-being but also researchers and practitioners interested in well-being assessment. We strongly believe that this journal is an important addition to the field of well-being assessment and that further advancement in the study of well-being is tied to the availability of high quality measures.

\section{Why a New Journal?}

The journal was founded to represent the growing needs for innovative and methodologically rigorous measurement tools in the rapidly growing research field on well-being. Furthermore, the worldwide expansion of well-being research calls for the development of fully tested translated versions of existing measures for use within single national contexts, as well as new well-being measures grounded into local traditions or applicable for international comparison. Moreover, new further developments in the study of well-being and the description of new constructs will require the development and evaluation of new assessment measures.

Research in well-being has been rapidly growing in recent decades. For example, Google Scholar generated 52.682 publications containing 'well-being' in the title (from 1950 to 2018), with $75 \%$ of them being published in the last 18 years (see Fig. 1). Half of all publications have been released in the past eight years (from 2011).

Ingrid Brdar

ibrdar@ffri.hr

Department of Psychology, Faculty of Humanities and Social Sciences, University of Rijeka, Rijeka, Croatia

2 Department of Psychology, Martin-Luther University Halle-Wittenberg, Halle, Germany

3 Department of Psychology, Faculty of Philosophy, University of Novi Sad, Novi Sad, Serbia 


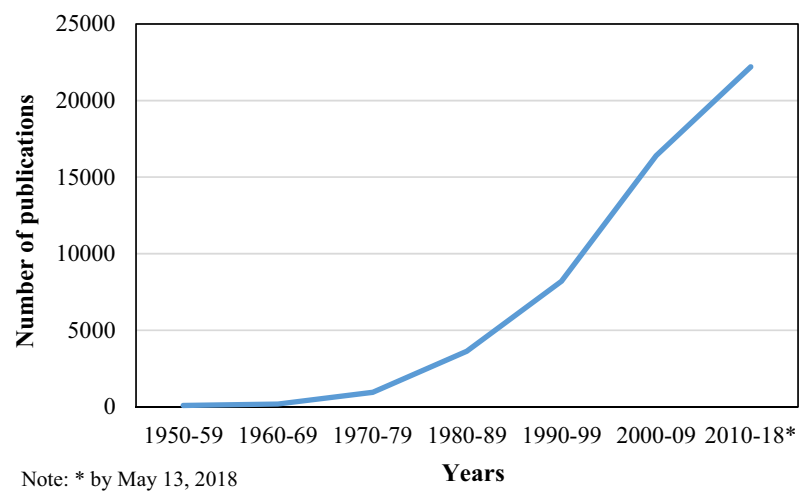

Fig. 1 The number of publication on well-being in the Google Scholar database (1950-2018)

Researchers are becoming increasingly aware that instrument validation is a critical part of research - especially, in the social sciences. The development of advanced statistical methods has raised standards for instrument validation. In addition, growing recognition of multiculturalism has raised interest for the development of new tests and instruments for international use. Hence, the need to analyze psychometric characteristics of measurement instruments has grown rapidly and the JWBA will be an outlet for these and related research efforts.

We explored the trend in publishing studies on well-being assessment in the PsycINFO database. The search terms were well-being and assessment (or related words - measurement, validation/validating, psychometric, factor structure, equivalence and invariance) in the abstract of articles published in peer-reviewed journals. The search produced nearly 221.794 articles (Fig. 2). Up to 1949, only 253 articles explored well-being assessment and validation, whereas 103.830 articles were published in the last period (2010-2018). After 1990, the number of articles considerably increased and continued to grow rapidly.

Researchers in social and behavioral sciences have recognized the need to test theoretical constructs across specific groups, populations, and cultures. These constructs are mostly measured by tests and questionnaires, predominantly developed in Western English-speaking

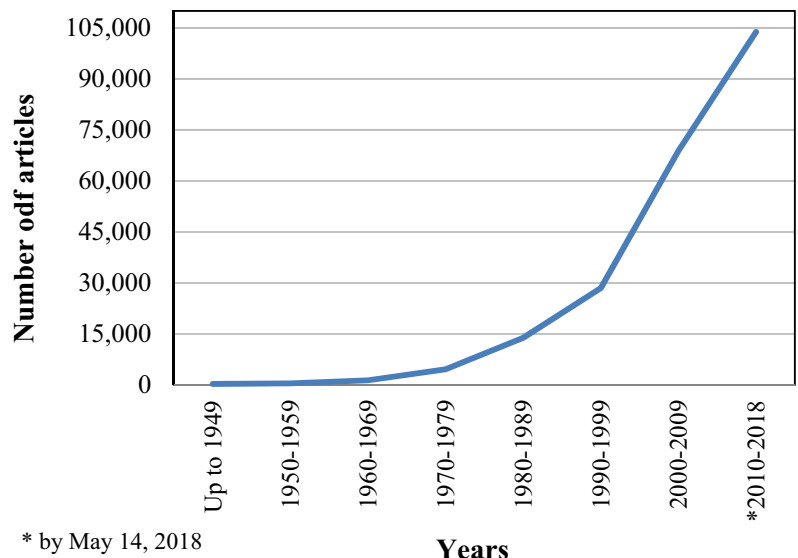

Fig. 2 The number of articles on well-being assessment and validation in the PsycINFO database 
countries. The growing number of multicultural studies has increased the need to adapt these measures for use in other than the source language. The time has come to set new standards for comparative studies of the assessment of well-being cross-culturally. In particular, researchers cannot just assume measurement invariance across groups - rather, such invariance can and must be tested.

Although researchers, particularly in cross-cultural research, have long recognized the importance of measurement equivalence in different groups (e.g., differing by age, gender), advanced statistical methods have been developed only in the last two decades and they are still evolving. For example, hierarchical (bifactor) exploratory structural equation modeling (ESEM) framework was recently introduced by Morin et al. (2016).

\section{About the Journal}

The journal is scheduled to publish three issues in a calendar year. In addition to the regular issues, the journal will periodically have special issues or sections dedicated to current problems or new emerging themes in the field of well-being assessment. Review articles are also welcome.

Specific topics covered by the JWBA include:

- development of new well-being assessment instruments, scales and methods

- validation of existing well-being measures and their adaptation for use within other cultural groups or contexts

- comparative studies testing measurement invariance in different groups and contexts (age, gender, or work organizations)

- integrative reviews of research in well-being assessment

Apart from the analysis of a measures' factor structure, contributions focused on the crosscultural adaptation of well-being measures should include additional evidence concerning the validity and possible application. All contributions are subject to peer review for scientific quality, originality and relevance to journal readership.

The intended audience of the journal includes scholars from disciplines involved in assessment, evaluation, testing, and other aspects of well-being measurement (psychology, sociology, health studies, social work, economics and related social science and behavioral disciplines). Furthermore, the journal will be of interest to practitioners concerned with the understanding and enhancing people's well-being (such as education, organizational psychologists, and health and social care organizations) and students attending courses in positive psychology, counseling and clinical psychology, organizational psychology, educational psychology, and methodology.

\section{Well-Being Assessment as a Broad Field: Conceptual Considerations}

Of course, there are many different conceptualizations and definitions of the term (subjective) well-being. The aim of the JWBA is to advance measurement of all of its different components and facets. For example, following the definition given by Diener et al. (1999) "Subjective well-being is a broad category of phenomena that includes people's emotional responses, 
domain satisfactions, and global judgments of life satisfaction" (p. 277), studies on the assessment of components of pleasant and unpleasant affect as well as components of life satisfaction and on domain satisfaction would be suitable for consideration in the journal.

It should be noted that the editors of the journal propose a broad view of well-being that is not limited to its emotional and cognitive components. For example, amongst other conceptualizations, submissions dealing with the measurement of psychological well-being (Ryff 1989), flourishing (e.g., Diener et al. 2010; Seligman 2011), mental health (Lamborn et al. 2017), happiness (Proyer et al. 2017), or hedonic and eudaimonic well-being (e.g., Peterson et al. 2005) fit the scope of the journal. In short, work on the assessment of variables that contribute to well-being in the sense of positive psychological functioning may be considered for publication. While the editors propose a comparatively broad view on what constitutes well-being, there are limitations to what can be considered. For example, other outlets will be better suited for the measurement of variables of correlates of well-being. For example, work on the measurement of negative functioning (with the potential exception of negative affectivity as a component of subjective well-being) is not a good fit for the journal. In this respect, we argue - in line with Jahoda (1958) - by saying that the absence of such problems may constitute a necessary, but not a sufficient criterion for well-being.

\section{Types of Samples}

The JWBA is open to work on the assessment of well-being in individuals of all age groups. We particularly encourage work on comparatively less well-studied groups such as younger children and elderly persons. We hope that stimulating more research on measurement issues in these groups will also facilitate more theoretical work and advance research on well-being as a whole. Additionally, the JWBA welcomes measurement instruments developed for the assessment of well-being in larger groups (e.g., measures of well-being in families or coworkers, etc.) and measurement issues related to work with existing data sets (e.g., panel data). In particular, we encourage work that contributes to a cross-cultural understanding of measurement issues such as language adaptations of existing instruments or comparisons of samples within one culture or across cultures (see e.g., Chang et al. 2017; Diener et al. 2003). In particular, the inclusion of data from non-Western, educated, industrialized, rich and democratic (non-WEIRD) societies is encouraged. Numerous studies come from the WEIRD societies, although they are among the least representative countries for generalizing research findings to all people and all cultures (Henrich et al. 2010).

Aside from papers describing newly developed instruments or adaptations to different languages, we also welcome basic research in the field. Examples may be proposals for new concepts (e.g., Schaufeli 2017), comparisons of existing measures (see e.g., Lucas et al. 1996), studies on challenges in the assessment of well-being in understudied groups (e.g., Macdougall et al. 2017; Shankar et al. 2017), the exploration of gender- or other relevant group-differences (e.g., Jiang and Huebner 2017), inter- and intraindividual fluctuations of well-being (e.g., Ong et al. 2007; Zheng et al. 2016), or on considerations related to the type of data collection mode (e.g., Weinberg et al. in press) — to name but a few. It should be noted that the JWBA is open to "initial" or "preliminary" studies in areas of great novelty, but has a particular focus that standards and common guidelines for the translation of research instruments are being followed (see e.g., Hambleton et al. 2005; van de van de Vijver and Hambleton 1996; van de van de Vijver and Tanzer 2004). For example, if a larger number of items in the original 
instruments get eliminated, but the authors do not provide a theoretically driven rational for doing so, cannot show that alternative translations and adaptations improve model fit, the work of adapting the instrument is not finished. Finally, the JWBA is also open to studies on the assessment of well-being in non-human samples as long as the submissions focus on measurement issues and provide a clear rationale for the methodology.

\section{Required Sample Characteristics}

Given the specialization of the journal, considerations around sample characteristics (e.g., size, distribution of scores, demographics, etc.) in empirical papers submitted to the JWBA are of particular interest. It is our understanding that for most submissions, one (small) student sample will not be sufficient for consideration for publication in the journal. While student samples are appropriate in some cases (i.e., those manuscripts dealing with the assessment of well-being in students), they will not suffice in many other cases. Additionally, many manuscripts will require analyses that are based on two or more independently collected samples (e.g., for replicating the proposed factor structure of a new measure). Of course, authors should consider recommendations for sample sizes for specific types of analyses and describe their consideration for the selection of their sample size.

\section{6 "Reliable and Valid"}

Many authors in the field of assessment described their measures as being "reliable and valid." However, such a statement is too broad for a journal focused on assessment such as the JWBA. Studies typically address selected facets of reliability and/or validity, while others are not explicitly tested. If authors report, for example, the Cronbach-alpha coefficient (or an alternative to $\alpha$ ), they can comment on the measures' internal consistency, but not on its "reliability" as a whole (e.g., its test-retest reliability). The same goes for the validity. Even reporting an elaborate Multi-Trait-Multi-Method design would not be sufficient for commenting on validity as a whole. Hence, clarity in the language is expected from the authors. Furthermore, authors are encouraged to address the reliability and validity of their instruments not only from a single, but also from multiple perspectives using a broad range of methods (see below). Prospective authors should also refrain from describing other measures (e.g., used for the validation of their measure) briefly as being "reliable and valid," but give detailed information on the type of reliability/validity assessed and the respective coefficients. This is in the interest of and contributes to the transparency of scientific work.

\section{Data Sources}

Prospective authors are encouraged to take advantage of the opportunity that different types of data sources provide. For example, it would considerably strengthen a study using questionnaires if the authors would also include findings from a peer-rating sample. In doing so, the robustness of the proposed associations beyond of what could critically be attributed to a joint method-bias could be examined (e.g., Campbell and Fiske 1959). Similarly, prospective authors are also encouraged to consider the inclusion of elaborate methods such as the Day 
Reconstruction Method (DRM) and the Experience Sampling Method (ESM) in their analyses. Overall, this should be understood as a plea to authors to work multi-methodically for improving the impact of their work whenever possible.

\section{Open Science Policy}

In light of recent discussions around the reproducibility of psychological studies, many efforts have been developed to increase the transparency of research with initiatives. Examples are recommendations to pre-register the study and to make the data and materials openly available to others. The editorial team of the JWBA supports these ideas and, therefore, we strongly encourage all authors to upload all data, materials, syntax, $R$-codes etc. either as online supplementary material and/or on websites such as the Open Science Framework (https://osf.io). The materials should allow for others to reproduce and verify the findings.

In the end, we want to thank all who have contributed to the new journal and to this inaugural volume. Thanks are due to many people who have helped in starting the JWBA. We would like to sincerely thank all authors and reviewers for their contributions. We greatly appreciate the support from the staff at Springer, in particular from Esther Otten, for her encouragement and assistance to set up the new journal. We are also grateful to Antonella Delle Fave and Filomena Maggino, the Editors in Chief of the Journal of Happiness Studies and Social Indicators Research, for their support in launching this journal.

It is truly a privilege and an honor to serve as the first Editors of the JWBA. We sincerely hope that you will find the journal interesting. We look forward to receiving your manuscripts and welcome your ideas and comments for making the journal highly impactful. Together we can build high-quality journal to serve the scientific community and practitioners in the field.

\section{References}

Campbell, D. T., \& Fiske, D. W. (1959). Convergent and discriminant validation by the Multitrait-Multimethod Matrix. Psychological Bulletin, 56(2), 81-105. https://doi.org/10.1037/h0046016.

Chang, E. C., Yang, H., Li, M., Duan, T., Dai, Y., Yang, J. Z., et al. (2017). Personal growth initiative and life satisfaction in Chinese and American students: Some evidence for using resources in the East and being planful in the West. Journal of Well-Being Assessment, 1(1-3). https://doi.org/10.1007/s41543-018-0004-2.

Diener, E., Oishi, S., \& Lucas, R. E. (2003). Personality, culture, and subjective well-being: Emotional and cognitive evaluations of life. Annual Review of Psychology, 54, 403-425. https://doi.org/10.1146/annurev. psych.54.101601.145056.

Diener, E., Suh, E. M., Lucas, R. E., \& Smith, H. L. (1999). Subjective well-being: Three decades of progress. Psychological Bulletin, 125(2), 276-302.

Diener, E., Wirtz, D., Tov, W., Kim-Prieto, C., Choi, D.-w., Oishi, S., \& Biswas-Diener, R. (2010). New wellbeing measures: Short scales to assess flourishing and positive and negative feelings. Social Indicators Research, 97(2), 143-156. https://doi.org/10.1007/s11205-009-9493-y.

Hambleton, R. K., Merenda, P., \& Spielberger, C. (Eds.). (2005). Adapting educational and psychological tests for cross-cultural assessment. Hillsdale, NJ: Lawrence Erlbaum Publishers.

Henrich, J., Heine, S. J., \& Norenzayan, A. (2010). The weirdest people in the world? Behavioral and Brain Sciences, 33(2-3), 61-83. https://doi.org/10.1017/S0140525X0999152X.

Jahoda, M. (1958). Current concepts of positive mental health. New York, NY: Basic Books.

Jiang, X., \& Huebner, S. E. (2017). Students' life satisfaction scale: Analysis of factorial invariance across gender. Journal of Well-Being Assessment, 1(1-3). https://doi.org/10.1007/s41543-017-0002-9. 
Lamborn, P., Cramer, K. M., \& Riberdy, A. (2017). The structural validity and measurement invariance of the Mental Health Continuum - Short Form (MHC-SF) in a large Canadian sample. Journal of Well-Being Assessment, 1(1-3). https://doi.org/10.1007/s41543-018-0007-z.

Lucas, R. E., Diener, E., \& Suh, E. (1996). Discriminant validity of well-being measures. Journal of Personality and Social Psychology, 71(3), 616-628.

Macdougall, H. K., O’Halloran, P., Sherry, E., \& Shields, N. (2017). Putting the athlete first: A comprehensive assessment of elite para athlete well-being. Journal of Well-Being Assessment, 1(1-3). https://doi. org/10.1007/s41543-017-0003-8.

Morin, A. J. S., Arens, A., \& Marsh, H. W. (2016). A bifactor exploratory structural equation modeling framework for the identification of distinct sources of construct-relevant psychometric multidimensionality. Structural Equation Modeling: A Multidisciplinary Journal, 23(1), 116-139.

Ong, A. D., Horn, J. L., \& Walsh, D. A. (2007). Stepping into the light: Modeling the intraindividual dimensions of hedonic and eudaemonic well-being. In A. D. Ong \& M. H. M. van Dulmen (Eds.), Oxford Handbook of Methods in Positive Psychology (pp. 12-25). New York, NY: OUP.

Peterson, C., Park, N., \& Seligman, M. E. P. (2005). Orientations to happiness and life satisfaction: The full life versus the empty life. Journal of Happiness Studies, 6(1), 25-41.

Proyer, R. T., Gander, F., Wellenzohn, S., \& Ruch, W. (2017). The Authentic Happiness Inventory Revisited: Addressing its psychometric properties, validity, and role in intervention studies. Journal of Well-Being Assessment, 1(1-3). https://doi.org/10.1007/s41543-018-0006-0.

Ryff, C. D. (1989). Happiness is everything, or is it? Explorations on the meaning of psychological well-being. Journal of Personality and Social Psychology, 57(6), 1069-1081.

Schaufeli, W. B. (2017). General Engagement: Conceptualization and Measurement with the Utrecht General Engagement Scale (UGES). Journal of Well-Being Assessment, 1(1-3). https://doi.org/10.1007/s41543-0170001-x.

Seligman, M. E. P. (2011). Flourish: A visionary new understanding of happiness and well-being. New York, NY: Free Press.

Shankar, S., Hubley, A. M., \& Zumbo, B. D. (2017). General, Health-specific, and housing-specific self-efficacy scales: Preliminary reliability and validity evidence with homeless or vulnerably housed adults. Journal of Well-Being Assessment, 1(1-3). https://doi.org/10.1007/s41543-018-0005-1.

van de Vijver, F. J. R., \& Hambleton, R. K. (1996). Translating tests: Some practical guidelines. European Psychologist, 1(2), 89-99.

van de Vijver, F. J. R., \& Tanzer, N. K. (2004). Bias and equivalence in cross-cultural assessment: An overview. European Review of Applied Psychology, 54(2), 11-135.

Weinberg, M.K., Cummins, R.A. Webb, D.A., \& Gwozdz, W. (in press). Incentivised online panel recruitment and subjective wellbeing: Caveat emptor. Journal of Well-Being Assessment.

Zheng, Y., Plomin, R., \& von Stumm, S. (2016). Heritability of intraindividual mean and variability of positive and negative affect: Genetic analysis of daily affect ratings over a month. Psychological Science, 27(12), 1611-1619. https://doi.org/10.1177/0956797616669994. 